\title{
A sensor for mechanical liquid properties utilizing pressure waves
}

\author{
Hannes Antlinger ${ }^{1}$, Roman Beigelbeck ${ }^{2}$, Samir Cerimovic ${ }^{3}$, Franz Keplinger ${ }^{3}$, Bernhard Jakoby ${ }^{1}$ \\ ${ }^{1}$ Institute for Microelectronics and Microsensors, Johannes Kepler University Linz, Altenberger Straße 69, \\ A-4040 Linz, Austria \\ 2 Institute for Integrated Sensor Systems, Austrian Academy of Sciences, Viktor Kaplan Straße 2, A-2700 \\ Wiener Neustadt, Austria \\ ${ }^{3}$ Institute of Sensor and Actuator Systems, Vienna University of Technology, Gußhausstraße 27-29, A- \\ 1040 Vienna, Austria
}

\begin{abstract}
This contribution presents the concept of a resonant sensor for fluid viscosity parameters. A lot of recent work has been focused on resonant sensors for, e.g., shear viscosity and mass density measurements. In this paper the concept of a sensor for the so-called "longitudinal viscosity" rather than the shear viscosity is introduced. We consider this parameter as equally suited for a lot of condition monitoring applications, in particular for liquids featuring higher viscosities. The associated theory including a simplified 1D model and present measurement results obtained with a first prototype device are outlined.
\end{abstract}

\section{Introduction}

Sensors for online monitoring of fluid properties facilitate characterization, controlling, and optimization of many processes. Requirements for these kinds of sensors are, e.g., small size, suitability for mass production, and robustness against environmental influences. Recently a lot of work has been focused on sensing viscosity of fluids (often combined with the mass density) which can be used as a measure for the state of the fluid to be monitored. A lot of this work is based on measurement principles using shear waves, e.g., [1].

In this contribution, we investigate an alternative resonant sensor concept based on viscous attenuation of pressure waves in a liquid. This device detects the so-called "longitudinal viscosity", i.e., a combination of shear viscosity and dilatational viscosity. Recently, other measurement setups using the principle of acoustic spectroscopy for the determination of the longitudinal viscosity have been presented [2], [3], where the attenuation of an acoustic pressure wave is determined as a function of the frequency. Compared to these principles, we aim at an integrated system utilizing a resonant sensor setup.

\section{Theory}

Our approach is based on monitoring the attenuation of pressure waves in a liquid sample. In order to achieve a higher sensitivity, the following resonant setup was chosen: Two planar, in-parallel rigid boundaries, separated by a distance $h$, form a channel where the liquid is enclosed. A transducer mounted on one boundary imposes resonating pressure waves in the liquid. The opposite boundary can be optionally equipped with a pressure sensor. Consequently, besides the impedance change of the transducer owing to the liquid loading, this signal can also be used as sensor signal as it will be mentioned below. The attenuation behavior of pressure waves in fluids is well known from the theory of acoustic wave propagation [4]. Fig. 1 depicts the basic sensor setup.

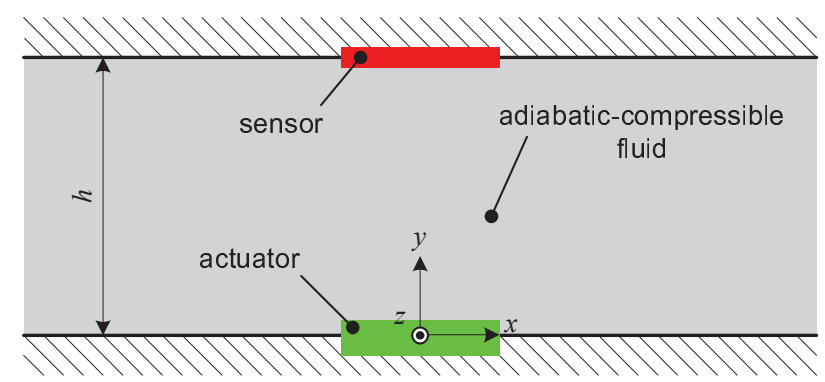

Fig. 1: Basic sensor setup. 
When focusing on viscous damping effects as sensor mechanism, both the first and also the second coefficient of viscosity ( $\mu$ and $\lambda$, respectively) need to be considered in detail. $\mu$ is the coefficient which represents the shear viscosity and is often only termed viscosity. $\lambda$ represents the dilatational viscosity associated with compressional stress components. Unfortunately, the definition of the second coefficient of viscosity is not consistent within the literature (see, e.g., [3], [4]), but our particular notation will become clear through the form of the Navier-Stokes equation stated below.

Based on the work in [5], a closed form solution for the pressure distribution in the spectral domain was derived by solving the linearized two-dimensional Navier-Stokes equation

$\rho_{0} \ddot{\mathbf{u}}=\frac{1}{\zeta_{S}} \nabla(\nabla \cdot \mathbf{u})+(\mu+\lambda) \nabla(\nabla \cdot \dot{\mathbf{u}})+\mu \nabla^{2} \dot{\mathbf{u}}$,

where $\mathbf{u}$ is the displacement vector, $\rho_{0}$ is the mass density, and $\zeta_{S}$ is the adiabatic compressibility. Introducing complex notation for the time-harmonic signals (time dependence $\exp (j \omega t)$ ) and applying the spatial Fourier transform

$$
\begin{aligned}
& P\left(k_{x}\right)=\int_{-\infty}^{\infty} p(x) e^{j k_{x} x} d x \\
& p(x)=\frac{1}{2 \pi} \int_{-\infty}^{\infty} P\left(k_{x}\right) e^{-j k_{x} x} d k x
\end{aligned}
$$

with respect to the $x$ coordinate, the closed-form solution for the pressure at the sensor plane $(y=h)$ in the spectral domain reads

$$
\begin{aligned}
& P\left(k_{x}\right)=\frac{U_{y 0}\left(k_{x}\right)\left[\frac{1}{\zeta_{s}}+j \omega(\lambda+2 \mu)\right] \xi_{1}\left(k_{x}^{2}-\xi_{3}^{2}\right)\left[\xi_{1} \xi_{3} \sinh \left(\xi_{1} h\right)-k_{x}^{2} \sinh \left(\xi_{3} h\right)\right]}{\left(k_{x}^{4}+\xi_{1}^{2} \xi_{3}^{2}\right) \sinh \left(\xi_{1} h\right) \sinh \left(\xi_{3} h\right)-2 k_{x}^{2} \xi_{1} \xi_{3}\left[\cosh \left(\xi_{1} h\right) \cosh \left(\xi_{3} h\right)-1\right]} . \\
& \xi_{1}=-\sqrt{k_{x}^{2}-\beta}, \xi_{3}=-\sqrt{k_{x}^{2}-\frac{\beta}{1+\alpha}}, \alpha=1+\frac{\lambda}{\mu}-\frac{j}{\omega \mu \zeta_{s}}, \beta=-j \omega \frac{\rho_{0}}{\mu}
\end{aligned}
$$

Here, $U_{y 0}\left(k_{x}\right)$ is the imposed normal displacement in the spectral domain at the actuator plane $(y=0), k_{x}$ is the spectral variable corresponding to $x$, and $\omega$ is the angular frequency.

When using a pressure sensor for generating the output signal and assuming that the output of this sensor corresponds to the pressure averaged over the sensing area, the value $P(0)=\int_{-\infty}^{\infty} p(x) d x$ can be used as approximation for the output signal (if we assume that the sensor covers the entire area with significant displacement amplitudes).

Using realistic material data $\left(\rho_{0}=1000 \mathrm{kgm}^{-3}, c_{0}=1500 \mathrm{~ms}^{-1}, \mu=10^{-3} \mathrm{Nsm}^{-2}, \lambda=0 \mathrm{Nsm}^{-2}, \omega=2 \pi \cdot 6 \cdot 10^{6} \mathrm{~s}^{-1}\right)$, a more detailed analysis of the associated terms leads to an approximate expression for $P\left(k_{x}\right)$ :

$$
P\left(k_{x}\right) \approx U_{y 0}\left(k_{x}\right)\left[\frac{1}{\zeta_{s}}+j \omega(\lambda+2 \mu)\right] \frac{\left(k_{x}^{2}-\xi_{3}^{2}\right)}{\xi_{3} \sinh \left(\xi_{3} h\right)} .
$$

This approximation is based on the numerically verified assumptions that $P\left(k_{x}\right) \approx 0$ for values of $k_{x}$ greater than $\pm\left|\xi_{3}(0)\right|,\left|\xi_{1}\right|>>\left|\xi_{3}\right|,\left|\xi_{1}\right| \approx$ const., and $k_{x-\max } \approx\left|\xi_{3}\right|_{\text {max }}$ in the relevant $k_{x}$ regions.

Eq. (4) reveals that the expression will always depend on $(\lambda+2 \mu)$ so that a separation of the two parameters is impossible even with non-uniform excitation and/or detection. Stokes' assumption, which says that $\mu$ and $\lambda$ are related as $\lambda=-2 / 3 \mu$, would enable a direct measurement of $\mu$. However, meanwhile it is well known that this assumption is invalid for most liquids [6]. Consequently, this sensing concept solely allows measurements of the longitudinal viscosity $(\lambda+2 \mu)$.

\section{Modeling and Simulation}

Since the setup basically determines the longitudinal viscosity, a simple 1D model for the sensor setup shown in Fig. 2 was implemented. Strictly, this model is only applicable if diffraction effects can be neglected (i.e., $h$ is smaller than the so-called Rayleigh distance characterizing the extension of the transducer's near field [7]). 


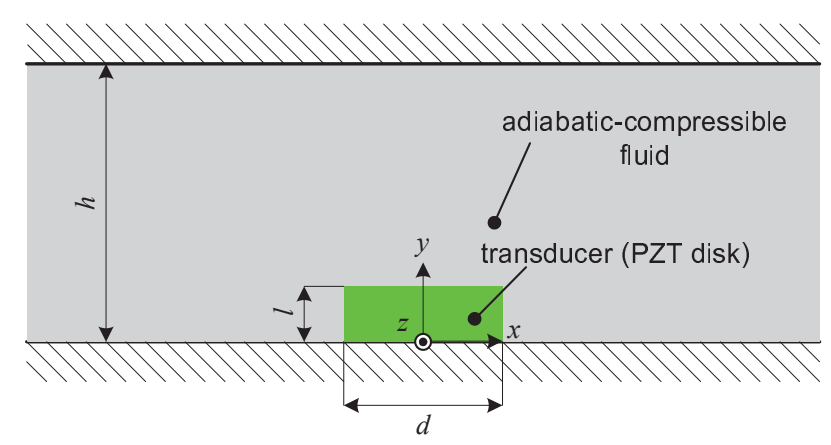

Fig. 2: Sensor setup utilizing a single PZT transducer.

The measurement setup consists of a PZT disk (diameter $d$, length $l$ ) acting as transducer and two rigid boundaries separated by a distance $h$. The piezo disk is modeled as a three port network with two acoustic ports and one electrical port, where a closed-form expression for the electric impedance as a function of the acoustic load impedances can be obtained [8]. The acoustic impedance $Z_{a c 1}$ represents the rigid boundary $\left(Z_{a c 1} \rightarrow \infty\right)$ where the PZT disk is mounted. The behavior of the fluid and the second boundary is modeled by an acoustic transmission line terminated with the acoustic impedance $Z_{L}\left(Z_{L} \rightarrow \infty\right.$ in case of a rigid boundary) featuring an acoustic input impedance $Z_{a c 2} . Z$ is the electrical input impedance of the piezoceramic transducer, which can be measured, e.g., with an impedance analyzer.

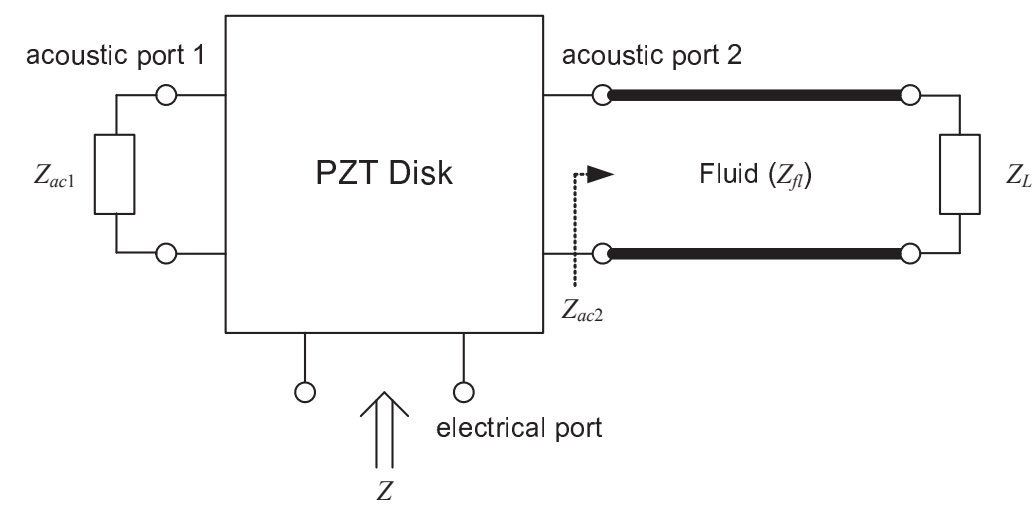

Fig. 3: 1D model for a setup utilizing a single PZT transducer.

The acoustic impedance is defined as $Z_{a c}=\frac{-T_{y y}}{v_{y}}$, where $T_{y y}$ is the $y y$-component of the stress tensor and $v_{y}$ is the velocity in $y$-direction [8]. With $T_{y y}=\frac{\partial u_{y}}{\partial y}\left[\frac{1}{\zeta_{s}}+j \omega(2 \mu+\lambda)\right]$ and $\frac{\partial T_{y y}}{\partial y}=-\rho_{f l} \omega^{2} u_{y}$ [5], the characteristic impedance $Z_{f l}$ and the propagation constant $k_{f l}$ of the "fluidic transmission line" are found as:

$$
\begin{aligned}
& Z_{f l}=\sqrt{\rho_{f l}\left(\frac{1}{\zeta_{s}}+j \omega(2 \mu+\lambda)\right)} \\
& k_{f l}=\frac{\omega \sqrt{\rho_{f l}}}{\sqrt{\frac{1}{\zeta_{s}}+j \omega(2 \mu+\lambda)}} .
\end{aligned}
$$

Similar to the theory of electrical transmission lines, the input impedance $Z_{a c 2}$ of a "fluid transmission line" terminated with an impedance $Z_{L}$ can be calculated as [9]:

$$
Z_{a c 2}=Z_{f l} \frac{Z_{L}+j Z_{f l} \tan \left(k_{f l}(l-h)\right)}{Z_{f l}+j Z_{L} \tan \left(k_{f l}(l-h)\right)} \text {. }
$$

Due to the resonating behavior of the mismatched transmission line, the intrinsic resonances of the piezo disk are superposed by the resonances of $Z_{a c 2}$. 
The quality factor of the resonances induced by the transmission line will depend on the attenuation of the acoustic waves in the liquid and thus also on $(\lambda+2 \mu)$. The dependence of the quality factor $Q$ on $(\lambda+2 \mu)$ for one resonance peak was studied by means of our model. We define $Q$ as $Q=f_{\text {res }} /\left(f_{2}-f_{1}\right)$ with $f_{\text {res }}$ as the frequency where a local maximum of $|Z|$ occurs. $f_{1}$ and $f_{2}$ are the two frequencies around the local maxima where $|Z|$ drops by $50 \%$ compared to the peak value at $f_{\text {res }}$. The full 1D simulation model described above was implemented in MATLAB. The following parameters have been used for the simulation:

PZT disk: $d=10 \mathrm{~mm}, l=1 \mathrm{~mm}$, Material PI-ceramic PIC-255, simulation data see [10].

Fluids (data taken from [11]):

$\begin{array}{lrccl} & \rho_{0}\left[\mathrm{kgm}^{-3}\right] & c_{0}[\mathrm{~m} / \mathrm{s}] & \mu\left[\mathrm{Nsm}^{-2}\right] & \lambda\left[\mathrm{Nsm}^{-2}\right] \\ \text { none (air) } & 1.161 & 343 & 18.600 \cdot 10^{-6} & 0 \\ \text { water } & 998.210 & 1497 & 1.002 \cdot 10^{-3} & 0 \\ \text { glycerine } & 1261.100 & 1904 & 945.000 \cdot 10^{-3} & 0\end{array}$

Geometry: $h=29 \mathrm{~mm}$.

Investigation of the quality factor $Q$ as a function of $(\lambda+2 \mu)$ reveals a significant drop of $Q$ in the high viscosity range. Fig. 4 shows the electrical impedance for air, water, and glycerine in the sample compartment. It can be seen, that comb-like resonances occur, which are associated with the resonances in the acoustic impedance $Z_{a c 2}$. According to the theory of transmission lines, the pattern repeats whenever the length of the line is increased by a half acoustic wavelength ${ }^{1}$ and thus its spacing is determined by the speed of sound in the fluid.
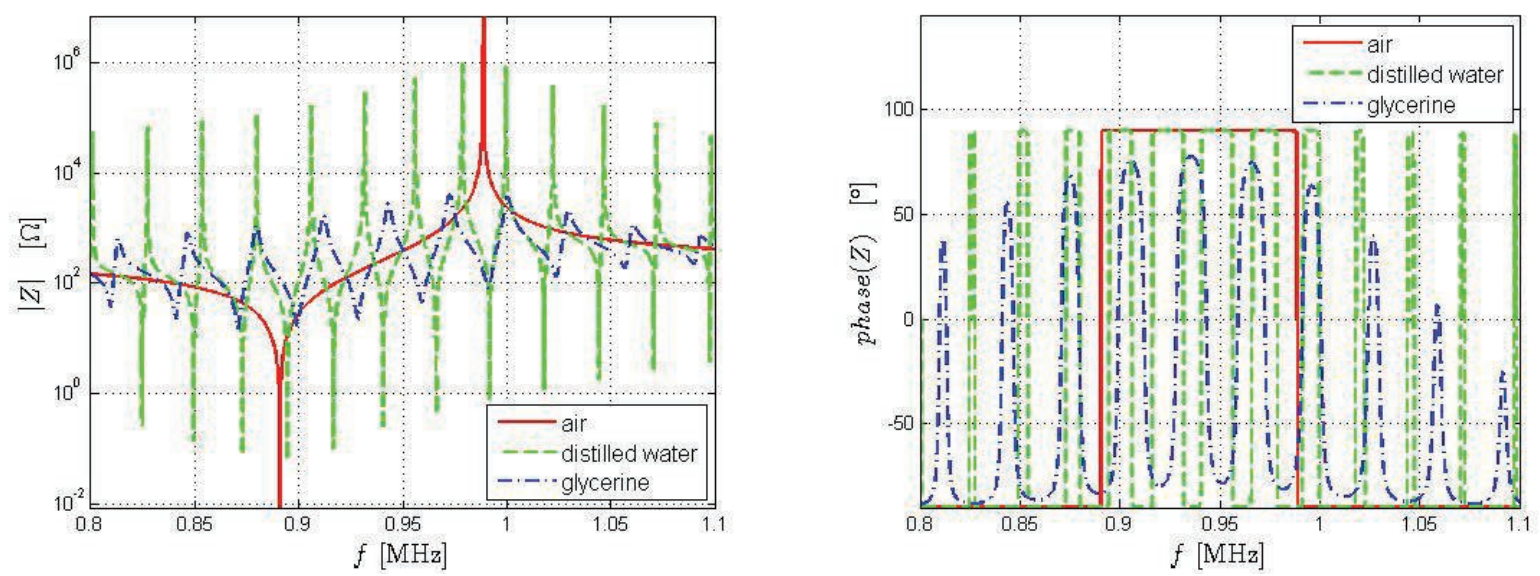

Fig. 4: Magnitude and phase of the piezo transducer for different media (simulation).

\section{Experimental results}

For first measurements, the prototype device shown in Fig. 5 was built. The walls are made of $1.5 \mathrm{~mm}$ FR4 material and carry two PIC255 piezo disks (diameter $d=10 \mathrm{~mm}$, length $l=1 \mathrm{~mm}$ ) each connected to an SMA connector. The distance $h$ (Fig. 2) is $29 \mathrm{~mm}$ for this device. For the following measurements only one of the PZT disk was used as transducer. The impedance was measured with an Agilent 4294 impedance analyzer.

Fig. 6 shows the magnitude and phase of the electrical impedance of the PZT transducer for the measuring cell filled with air, distilled water, and glycerine. It turned out that it is not possible to calculate $Q$ as defined above since the intrinsic damping of the setup (including the intrinsic damping of the PZT disks, damping due to adhesives and protection layers) is superposed to that caused by the viscous attenuation. Thus the $Q$-factor associated with viscous attenuation cannot be extracted using the simple definition above. We are currently working on a refined model yielding the $Q$-factor considering only the viscously induced damping. However, the qualitative behavior predicted by our model can be observed: comb-like resonances (with frequency spacing corresponding to the resonating pressure waves just as in

\footnotetext{
${ }^{1}$ Strictly this holds for lossless lines only.
} 
our model) can be observed, where the quality factor (indicated by width and height of each individual resonance) decreases with increasing viscosity².

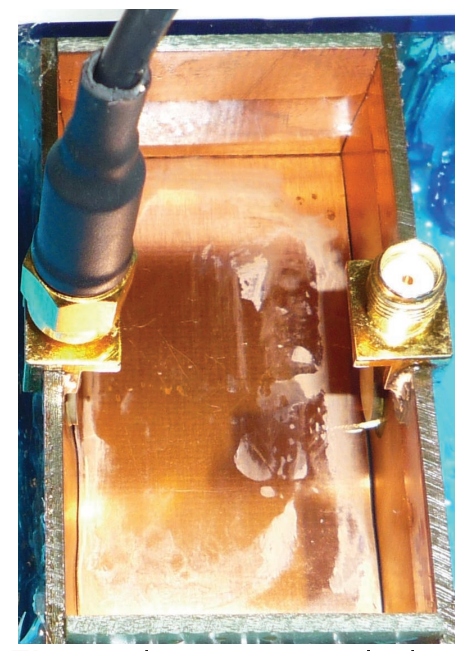

Fig. 5: First prototype device

Further discrepancies between model and simulation include a frequency shift of the resonances associated with the PZT transducer. It appears as if the walls cannot be approximated as rigid walls but rather resemble free space which is near at hand if one considers that the acoustic impedance of FR4 can be expected to be comparatively low.
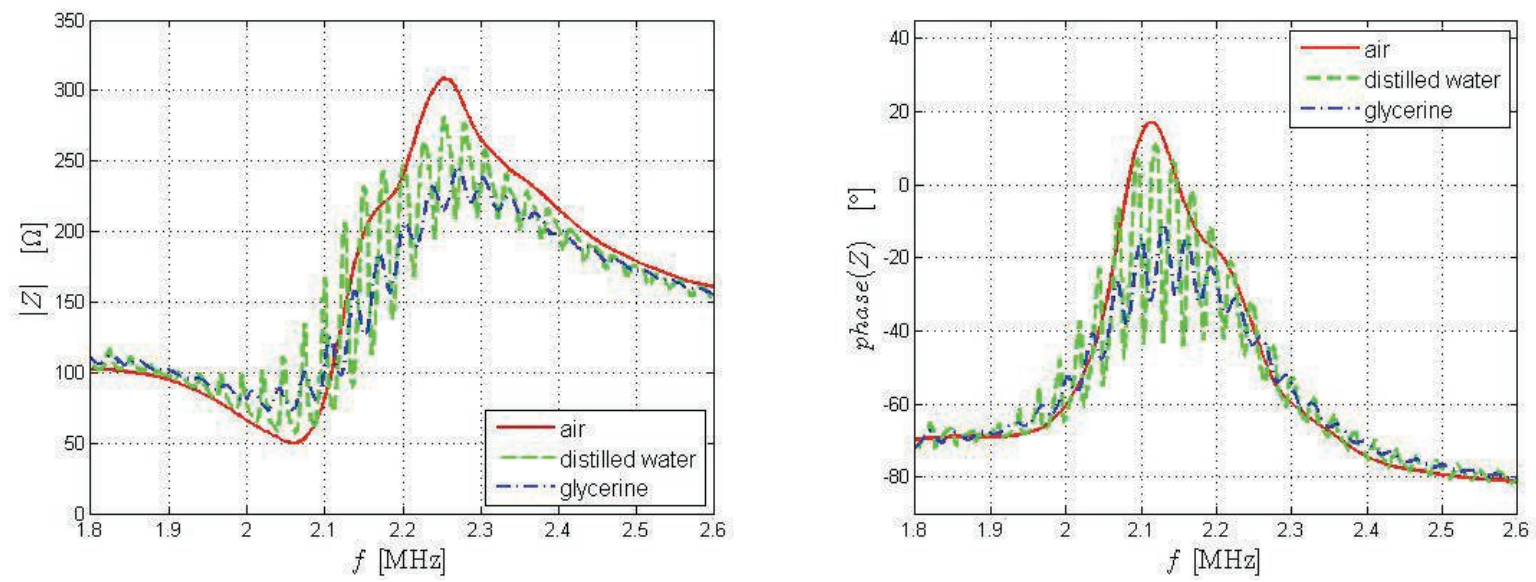

Fig. 6: Magnitude and phase of the piezo transducer with different media (experiment).

\section{Conclusions}

In this paper we have presented a possibility for measuring the so-called "longitudinal viscosity" using a resonant setup. First measurements show that a clear dependence of the electrical impedance on the fluid parameters can be obtained, in particular the damping of the observed resonances is related to the (longitudinal) viscosity and the frequency spacing to the sound velocity. A simple model has been devised. It enables qualitative predictions of the experimentally observed behavior but needs further refinements to account for acoustic losses in the setup as well as for imperfections in the walls of the resonators (which do not act as acoustic open circuits). Future research will deal with a refined analysis and improvements of the prototype device.

\section{Acknowledgements}

This work was supported by the Austrian Science Fund (FWF), grant L657-N16.

\footnotetext{
${ }^{2}$ Note that the values for the second coefficients of viscosity for most liquids are not securely established yet, however, it is a safe bet to assume that glycerine features a higher longitudinal viscosity than water.
} 


\section{References}

[1] B. Jakoby, R. Beigelbeck, F. Keplinger, F. Lucklum, A. Niedermayer, E. Reichel, C. Riesch, T. Voglhuber-Brunnmaier, and B. Weiss, "Miniaturized sensors for the viscosity and density of liquids performance and issues," IEEE Trans. on Ultrason., Ferroelec., and Freq. Contr., vol. 1, no. 57, pp. 111120, 2010.

[2] M.J. Holmes, N.G. Parker and M.J.W. Povey, "Temperature dependence of bulk viscosity in water using acoustic spectroscopy," Journal of Physics: Conference Series, Proceedings of the AngloFrench Physical Acoustics Conference, January 2010, arXiv:1002.3029v1 [physics.flu-dyn].

[3] Andrei S. Dukhin and Philip J. Goetz, "Bulk viscosity and compressibility measurement using acoustic spectroscopy," J. Chem. Phys. 130, 124519 (2009).

[4] L.D. Landau, E.M. Lifshitz, Fluid Mechanics, Second Edition: Volume 6 (Course of Theoretical Physics), Butterworth-Heinemann, 1987.

[5] Beigelbeck, R., and Jakoby, B. "A two-dimensional analysis of spurious compressional wave excitation by thickness-shear-mode resonators," Journal of applied physics, 95(9):4989-4995, May 2004.

[6] L.N. Liebermann, "The second viscosity of liquids," Phys. Rev., vol. 75, nr. 9, p- 1415-1422, 1949.

[7] Lerch, R., Sessler G., Wolf, D., Technische Akustik, Grundlagen und Anwendungen, Springer Verlag 2009.

[8] Kino, Gordon S., Acoustic waves: devices, imaging, and analog signal processing, Prentice-Hall, Inc. 1987.

[9] D.M. Pozar, Microwave Engineering, Addison-Wesley, 1993.

[10] PI-ceramic, PIC255 material coefficients data, http://www. piceramic.com.

[11] David R. Lide, CRC Handbook of chemistry and physics 86th edition 2005-2006. 